\title{
Role of lymphadenectomy in clinically organ-confined prostate cancer
}

\author{
Nivedita Bhatta Dhar • Fiona C. Burkhard • \\ Urs E. Studer
}

Received: 9 January 2007 / Accepted: 14 January 2007 / Published online: 14 February 2007

(C) Springer-Verlag 2007

\begin{abstract}
There has been considerable debate about the utility of pelvic lymph node dissection (PLND) when performing a radical prostatectomy. Reported practices vary from those who always perform an extended PLND to those who employ a predictive nomogram in their decision making to those who are increasingly not performing a PLND in low-risk disease. A Medline search was used to identify relevant manuscripts dealing with the role of lymphadenectomy in clinically organ-confined prostate cancer. A greater number of lymph nodes (LN) removed and examined at prostatectomy for prostate cancer appears to increase the likelihood of finding LN metastases and increase prostate cancer-specific survival even in patients who have histologically uninvolved LN. This survival benefit may result from more accurate staging and possible removal of occult metastases. The need for and extent of PLND in prostate cancer, especially in low-risk disease, however, is unlikely.
\end{abstract}

Keywords Prostate cancer - Lymphadenectomy • Nodal metastases $\cdot$ Radical prostatectomy

\section{Introduction}

The incidence of nodal metastases diagnosed during radical prostatectomy (RP) has declined in recent years, related to the downward pathologic stage migration induced by prostate-specific antigen (PSA)-based

N. Bhatta Dhar · F. C. Burkhard · U. E. Studer $(\bowtie)$

Department of Urology, University of Bern,

Inselspital, Bern, Switzerland

e-mail: urology_berne@insel.ch screening [1, 2]. Lymph node (LN) metastases in clinically localized prostate cancer portends a poor prognosis [3]. Therefore, algorithms that include preoperative risk factors (serum PSA level, clinical stage, and tumor grade) have since been introduced to identify patients at low risk of nodal metastasis and in whom pelvic lymph node dissection (PLND) may not be beneficial [4-6]. For prostate cancer patients with a Gleason sum of 6 or less and a PSA level of $10 \mathrm{ng} / \mathrm{ml}$ or less, the likelihood of metastatic disease according to the Partin nomogram is $0-3 \%$ [7]. For this reason, and consistent with most other nomogram predictions, some surgeons reserve PLND for men with a PSA level greater than $10 \mathrm{ng} / \mathrm{ml}$ and Gleason sum greater than 6 [8]. However, these data are based on experience with a limited PLND dissection with few LNs being histopathologically analyzed resulting in an underestimate of the true pathological stage and incidence of positive nodes. Therefore, the utility of such nomograms have been limited by differences in sensitivity to discriminate between low-risk and high-risk patients [9].

\section{Clinical staging}

Accurate staging is important, not only for identification of the extent and location of the malignancy but also, perhaps more importantly, for determination of malignant potential. In prostate cancer, despite advances in radiological examinations, computed tomography (CT) and magnetic resonance imaging (MRI) have not proven to be sufficient for detection of LN metastasis in the pelvis $[10,11]$.

In recent years the sentinel lymph node (SLN) concept has been applied to various malignant tumors. 
SLN, a term introduced by Cabanas, denotes the LN where lymphatic flow from the tumor first arrives [12]. According to the pioneering works of Wawroschek et al. it was possible to identify SLNs in patients with prostate cancer with the radioisotope method [13]. By using a modified PLND in 117 patients, the incidence of LN metastasis was consistent with that of SLNs in 26 of 27 patients with LN metastasis, thus demonstrating the high sensitivity rate of $96 \%$ [13].

Recently, it was also observed that the combination of SLN lymphocintigraphy and extended PLND removes more positive nodes than SLN lymphocintigraphy alone [14].

As stated earlier, the use of MRI and CT scan to evaluate LN involvement is not routinely recommended, owing to the low sensitivity $(0-30 \%)$ in imaging microscopic disease [15]. Anatomic localization of prostatic SLN with fusion imaging of SPECT and CT scans after intraprostatic injection of Technetium-99m-Nanocolloid identified 317 SLN with a median of 10 per patient (range 3-19) [16]. But since the objective is to not only identify diseased nodes but also to remove as many as possible, the role of preoperative SLN detection remains undetermined. Specialized techniques, such as high-resolution MRI used in tandem with the intravenous administration of lymphotropic superpara-magnetic nanoparticles, might allow for detection of small and otherwise undetectable $\mathrm{LN}$ disease. In a recent study involving 80 patients with clinical T1, T2, or T3 disease, MRI with lymphotropic superpara-magnetic nanoparticles outperformed conventional MRI and nomograms in the detection of LN-positive disease [17]. An issue of concern, is that nanoparticles do not adequately identify nodes with micrometatases yet it is these patients who are most likely to benefit from PLND. Therefore such novel imaging techniques require further clinical evaluation and validation before widespread use.

Monoclonal antibody radioimmunoscintigraphy (i.e., ProstaScint Scan; Cytogen Corporation, Princeton, NJ, USA) has had limited accuracy in the detection of LN metastases because the antibody targets an intracellular epitope that is only exposed in dying or dead cells [18]. Although initially promising, molecular techniques using reverse transcription polymerase chain reaction have had varying sensitivities in detecting circulating cancer cells. In addition, a significant proportion of men with organ-confined disease in one study were found to have a positive PSA PCR assay [19]. Thus, the significance of a positive assay remains unknown, and positive assays might lead to men being over staged and denied curative treatment.
In continued efforts to improve patient outcomes and to tailor treatment options to individual patient circumstances, predictive nomograms that estimate the likelihood of positive nodes for an individual by assigning points for specific risk factors have been developed for predicting pathologic stage and biochemical failure after definitive therapy. However as stated before, these nomograms are based on limited PLND and therefore may be imprecise. Recently, Briganti et al. developed and validated the first nomogram predicting the probability of LN invasion that accounts for the number of nodes removed and examined in patients undergoing PLND of various extents [20]. Based on their model, the risk of LN invasion for men undergoing RRP increases linearly in proportion to the number of $\mathrm{LN}$ removed. This nomogram was internally validated and was $76 \%$ accurate. While identifying new factors that add incremental predictive accuracy obviously helps, the above nomogram depends on its development cohort. Primary lymphatic drainage sites of the prostate may go up to the inferior mesenteric artery but Briganti and colleagues did not account for this in their nomogram. In addition, nomogram predictions must be interpreted as such; they do not make treatment recommendations or provide definitive information on disease progression or complications associated with treatment.

\section{PLND template}

The actual definition of limited/extended PLND for prostate cancer is variable. The minimal PLND considers only the obturator fossa; the standard variant also includes LN along the external iliac vein, whereas the extended variant is a complete $\mathrm{LN}$ dissection along the obturator fossa and the external, internal, and, in some cases, common iliac vessels. As determined by cadaveric and human studies, the average number of nodes obtained by extended PLND is 22 [21].

\section{Pelvic lymphadenectomy as a staging procedure}

Uniform surgical standards of pelvic staging lymphadenectomy for prostate cancer cannot be determined from the current literature. The rationale for locoregional staging lymphadenectomy in prostate cancer lies in the accurate diagnosis of occult micrometastases in order to stratify patients who might benefit from adjuvant therapeutic measures. One study has shown that early androgen-ablative therapy following $\mathrm{RP}$ in men with node-positive disease is associated with 
improvements in survival [22]. Therefore, an exact LN staging could be helpful to select patients for the best adjuvant treatment. Furthermore, the dissection of LN (micro)metastases could improve survival.

A limited PLND is not a reliable staging procedure because it misses $>50 \%$ of the positive nodes compared to an extended dissection. Extended PLND has been shown to increase the yield of both total LN and LN metastases significantly. Heidenreich et al. reported on 103 patients who had an extended PLND comprising the external iliac, internal iliac, obturator, and common iliac LN bilaterally and the presacral nodes [23]. They compared this group of patients with 100 men who received only standard PLND. LN metastases were diagnosed in $26.2 \%$ in the extended group and in only $12 \%$ in the standard PLND group. Despite negative obturator LN, positive LN were identified in the internal iliac and presacral regions. Forty-two percent of all LN metastases were detected outside the regions of standard PLND. Bader et al. performed a meticulous PLND along the external iliac vein, the obturator nerve, and the internal iliac (hypogastric) vessels in men with clinically organ-confined disease. Eightyeight of 365 men (24\%) had positive LN. Internal iliac $\mathrm{LN}$ were positive in $58 \%$ and internal iliac $\mathrm{LN}$ alone were positive in $19 \%$ of the men [24]. Similar results have been reported using the laparoscopic approach. Stone et al. reported twice as many LN removed via extended than limited laparoscopic PLND (mean 17.8 versus 9.3), and three times as many patients with $\mathrm{LN}$ metastasis in the extended group [7.3\% versus $23.1 \%$ $(P=0.02)]$ [25]. However, the link between the extent and the yield of PLND was not always confirmed. The only randomized study on extended versus limited PLND was reported by Clark et al. found no difference in the yield of positive nodes with more extended dissection (extended 3.2\% versus limited 2.4\%) [26]. The two types of dissection were conducted in the same patient on contralateral sides, however, the majority of patients had a low probability of LN metastases and thereby did not require a PLND. Comparing two techniques in a cohort who are unlikely to have positive $\mathrm{LN}$ obviously limits the power of the trial. Even without this limitation, the number of patients examined was inadequate for an equivalence study. In addition, $90 \%$ of their patients had T1c or T2a disease, which is mostly a unilateral disease, and therefore randomly assigning them to extended PLND on only one side carries a substantial risk that the extended PLND was performed on the nontumor bearing side. Also, neither the number of nodes removed, nor the pathological specimen assessment were defined, which would have introduced an important bias. Therefore, the study design renders it hard to compare their data with those of other previous studies.

Taken together, the above data suggest that, in general, extended PLND yields higher rates of LN. A possible reason for the difference in the proportion of positive nodes found at limited and extended PLND is that approximately one fifth of the patients with positive nodes found at extended PLND have the internal vessels as their sole site of disease [19, 20]. A preferred anatomical location for positive nodes has not been identified. Since prostate cancer nodal metastases do not follow a pre-defined pathway of metastatic spread, an extended PLND identifies LN invasion that would not otherwise be detected by a limited PLND. Importantly, the above studies demonstrate that up to two thirds of all patients with LN metastasis have positive nodes along the internal iliac vessels, an area not included in a limited PLND.

\section{A therapeutic benefit of extended lymphadenectomy}

Extended lymphadenectomy enhances the accuracy of surgical staging but if this translates into a survival benefit has yet to be established. Indeed, the limited value of a PLND as a staging procedure only without any therapeutic benefit is currently increasingly challenged [27]. The possibility of therapeutic benefit for PLND in prostate cancer has been suggested by some studies.

Some studies show that patients with minimal LN metastases will have better prognosis by removing the diseased nodes. In 1987, Golimbu et al. retrospectively analyzed 42 patients with occult nodal disease who underwent PLND and RRP [28]. In this series, patients with low tumor bulk and one positive $\mathrm{LN}$ had survival rates comparable to those of matched controls after a mean follow-up of 5 years. In 1988, Catalona showed that treatment can be curative even in $\mathrm{LN}$-positive disease. In a relatively small series of 12 patients with $\mathrm{LN}$ involvement and no adjuvant therapy, $75 \%$ remained recurrence free at 5 years and $58 \%$ at 7 years [29]. The results from Pound et al. for patients with $\mathrm{LN}$ micrometastasis, revealed a 10-year metastasis-free survival rate of $68 \%$, again without adjuvant therapy [30]. In 2003, Bader et al. reported on 92 men followed with histologically proven LN metastases who received no adjuvant therapy. After 45 months (median), 15 of 39 patients $(38.5 \%)$ with only one positive node remained without signs of progression, whereas only 10 and $14 \%$ of patients with two or more positive LN remained disease free [31].

Prognosis of patients has also been shown to be depend on the number of positive nodes involved. 
Daneshmand et al. reported that patients who had one or two positive LNs had a clinical recurrence-free survival rate of 70 and $73 \%$ at 10 years [32]. By contrast, men with five or more involved nodes had a recurrence-free survival rate of only $49 \%$. When stratified by LN density, patients with a density of $\geq 20 \%$ were at higher risk of clinical recurrence than those with a density of $<20 \%$. A difference in PSA progression-free survival in men with $<15 \%$ of nodes involved was reported by Allaf et al. [33]. In these patients, the 5year PSA progression-free survival was $43 \%$ for the extended and only $10 \%$ for the limited PLND.

Recently, Bader et al. demonstrated that the number of LN metastases detected is strictly correlated to the number of nodes removed and that the rate of $\mathrm{pN} 0$ patients with tumor progression is higher in patients with only few nodes removed [34]. Similarly, Joslyn et al., using the SEER database, concluded that patients with LN involvement had a significantly greater number of nodes removed compared with those with no LN involvement and that extended PLND reduces the long-term risk of prostate cancerrelated death, even in patients with negative nodes compared to patients without PLND [35]. One of the limitations posed by using SEER data is the lack of information regarding adjuvant hormonal therapy. A potential explanation for these observations is that a thorough nodal resection eliminated micrometastases that were not detected by routine histologic examination. Recently, Masterson et al. examined the association between the number of LNs removed, the number of positive LNs and disease progression in patients undergoing PLND and RRP for clinically localized prostate cancer [36]. Of the 4,611 eligible patients a median of 9 LNs were removed and positive nodes were found in 175 patients $(3.8 \%)$. Overall the number of LNs removed did not predict freedom from biochemical recurrence (BCR), however, in men without nodal involvement an increased number of nodes removed correlated significantly with freedom from $\mathrm{BCR}$. Recently, an assay that reveals and quantifies clinically relevant occult micrometastases in pathologically negative $\mathrm{LN}$ at the time of primary prostate cancer therapy was developed [37]. The prognostic importance of pathologically detected LN metastases been will established by markedly inferior final outcome but the new question is whether occult micrometastatic in pathologically N0-LN might also be associated with a reduced prognosis [3].

The above investigators concluded that a significant benefit in BCR-free survival might exist for certain subgroups undergoing the extended dissection. Nevertheless, it is unlikely that men with high-risk disease will be cured by removing all positive nodes. But, it is possible that progression-free survival can be prolonged when radical surgery and adjuvant therapy are combined [38]. One possible explanation for the finding of a benefit to extended dissection is that it might be an artifact of stage migration (the Will Rogers phenomenon) [39]. A limitation of many such studies is that the proportion of patients with prostate cancer with positive nodes is low, and therefore the value of extended PLND requires a multi-institutional, randomized clinical trial.

By contrast to the above studies, DiMarco et al. could not confirm that the extent of PLND affected prostate cancer outcome in LN-negative men [40]. They concluded that the effect of understaging on outcome is likely to be negligible. As they compared the results of extended PLND between 1987 and 1989 with those of a more limited PLND from 1999 to 2000, Tstage migration, with higher tumor stages in the earlier period, could explain these results. Because an extended PLND in the earlier period led to similar results for disease progression and survival as removing fewer nodes in a later period, their results imply that the extended PLND had a therapeutic role for the earlier cohort.

Although these data suggest the benefit of extended PLND at prostatectomy, some argue that it would be hard to advocate that approach in all patients, particularly those with low risk of $\mathrm{LN}$ involvement. The need for and extent of PLND in prostate cancer, especially in low-risk disease, is unlikely. Bhatta Dhar et al. reported a 6-year biochemical relapse-free rate for the PLND versus no-PLND group of 86 and $88 \%$, respectively and on multivariate analysis, PLND was not an independent predictor of outcome $(P=0.33)$. Recently Schumacher et al. reported that with an extended PLND in 231 patients with a median serum PSA of $<10 \mathrm{ng} / \mathrm{ml}$ (range $0.4-9.98$ ), most positive nodes were found in the subgroup of patients with a Gleason score $\geq 7$ in the surgical specimen $(25 \%)$, whereas in patients with a Gleason score $\leq 6$ only $3 \%$ had positive nodes [41]. They concluded that the incidence of LN metastasis is low in patients with a PSA $<10 \mathrm{ng} / \mathrm{ml}$ and Gleason score $\leq 6$ and in these patients PLND may be unnecessary. However, there is a substantial risk of preoperative understaging and undergrading and this must be considered when counseling patients. Grossfeld et al. found $30 \%$ undergrading and understaging in patients with a preoperative biopsy Gleason score $\leq 6$ [42].

Opponents of extended PLND argue that performing an extended PLND results in increased morbidity and higher costs [43]. Morbidity can be kept low (approx. 2\% lymphoceles) if attention is paid to a few 
details: (1) Ligation of lymphatic vessels coming from the legs, instead of clipping. Hemoclips have tendency to be torn away during subsequent surgery. (2) Placement of two drains one on each side of the pelvis where PLND was performed. Drains should be removed gradually until the total amount drained is less than $50 \mathrm{ml} / 24 \mathrm{~h}$. (3) Injection of low molecular heparin into the upper arm instead of the thigh.

In conclusion, a greater number of $\mathrm{LN}$ removed and examined at prostatectomy for prostate cancer appears to increase the likelihood of finding LN metastases and increase prostate cancer-specific survival even in patients who have histologically uninvolved LN. This survival benefit may result from more accurate staging and possible removal of occult metastases.

\section{References}

1. Danella JF, deKernion JB, Smith RB et al (1993) The contemporary incidence of lymph node metastases in prostate cancer: implications for laparoscopic lymph node dissection. J Urol 149:1488-1491

2. Petros JA, Catalona WJ (1992) Lower incidence of unsuspected lymph node metastasis in 512 consecutive patients with clinically localized prostate cancer. J Urol 147:1574-1575

3. Gervasi LA, Mata J, Easley JD et al (1989) Prognostic significance of lymph nodal metastases in prostate cancer. J Urol 142:332-336

4. Partin AW, Mangold LA, Lamm DM, Walsh PC, Epstein JI, Pearson JD (2001) Contemporary update of prostate cancer staging nomograms (Partin tables) for the new millennium. Urology 58(6):843-848

5. Narayan P, Fournier G, Gajendran V et al (1994) Utility of preoperative serum prostate specific antigen concentration and biopsy Gleason score in predicting risk of pelvic lymph node metastases in prostate cancer. Urology 44:519-524

6. Bluestein DL, Bostwick DG, Bergstralh EJ et al (1994) Eliminating the need for bilateral pelvic lymphadenectomy in select patients with prostate cancer. J Urol 151:1315-1320

7. Partin AW, Kattan MW, Subong EN et al (1997) Combination of prostate-specific antigen, clinical stage, and Gleason score to predict pathological stage of localized prostate cancer. A multi-institutional update. JAMA 277:1445-1451

8. Cagiannos I, Karakiewicz P, Eastham JA et al (2003) A preoperative nomogram identifying decreased risk of positive pelvic lymph nodes in patients with prostate cancer. J Urol 170(5):1798-1803

9. Augustin H, Eggert T, Wenske S et al (2004) Comparison of accuracy between the Partin tables of 1997 and 2001 to predict final pathological stage in clinically localized prostate cancer. J Urol 171:177-181

10. Wolf JS Jr, Cher M, Dall'era M, Presti JC Jr, Hricak H, Carroll PR (1995) The use and accuracy of cross-sectional imaging and fine needle aspiration cytology for detection of pelvic lymph node metastases before radical prostatectomy. J Urol 153(3 Pt 2):993-999; review

11. Tempany CM, McNeil BJ (2001) Advances in biomedical imaging. JAMA 285(5):562-567

12. Cabanas RM (1977) An approach for the treatment of penile carcinoma. Cancer 39:456
13. Wawroschek F, Vogt H, Weckermann D, Wagner T, Hamm M, Harzmann R (2001) Radioisotope guided pelvic lymph node dissection for prostate cancer. J Urol 166:1715

14. Weckermann D, Dorn R, Holl G, Wagner T, Harzmann R (2006) Limitations of radioguided surgery in high-risk prostate cancer. Eur Urol 11 Sep 2006 (epub ahead of print)

15. Borley N, Fabrin K, Sriprasad S et al (2003) Laparoscopic pelvic lymph node dissection allows significantly more accurate staging in "high-risk" prostate cancer compared to MRI or CT. Scand J Urol Nephrol 37:382-386

16. Mattei A et al (2006) Anatomic localization of prostatic sentinel lymph nodes (SLN) according to fusion imaging of SPECT and CT scans after intraprostatic injection of Technetium-99m-Nanocolloid. J Urol 175(S4):448, Abstract 1388

17. Harisinghani MG, Barentsz J, Hahn PF et al (2003) Noninvasive detection of clinically occult lymph-node metastases in prostate cancer. N Engl J Med 348:2491-2499

18. Troyer JK, Beckett ML, Wright GL Jr (1997) Location of prostate-specific membrane antigen in the $\mathrm{LN} \mathrm{CaP}$ prostate carcinoma cell line. Prostate 30:232-242

19. Katz AE, Olsson CA, Raffo AJ et al (1994) Molecular staging of prostate cancer with the use of an enhanced reverse transcriptase-PCR assay. Urology 43:765-775

20. Briganti A, Chun FK, Salonia A, Gallina A, Farina E, Da Pozzo LF, Rigatti P, Montorsi F, Karakiewicz PI (2006) Validation of a nomogram predicting the probability of lymph node invasion based on the extent of pelvic lymphadenectomy in patients with clinically localized prostate cancer. BJU Int 98(4):788-793

21. Weingartner A, Ramaswamy A, Bittinger A et al (1996) Anatomical basis for pelvic lymphadenectomy in prostate cancer results of an autopsy study and implications for the clinic. J Urol 156:1969-1971

22. Messing EM, Manola J, Yao J, Kiernan M, Crawford D, Wilding G, di'SantAgnese PA, Trump D; Eastern Cooperative Oncology Group study EST 3886 (2006) Immediate versus deferred androgen deprivation treatment in patients with node-positive prostate cancer after radical prostatectomy and pelvic lymphadenectomy. Lancet Oncol 7(6):472-479

23. Heidenreich A, Varga Z, von Knobloch R (2002) Extended pelvic lymphadenectomy in patients undergoing radical prostatectomy: high incidence of lymph node metastasis. J Urol 167:1681-1684

24. Bader P, Burkhard FC, Markwalder R, Studer UE (2002) Is a limited lymph node dissection an adequate staging procedure for prostate cancer? J Urol 168:514-518

25. Stone NN, Stock RG, Unger P (1997) Laparoscopic pelvic lymph node dissection for prostate cancer: comparison to the extended and modified techniques. J Urol 158:1891-1894

26. Clark T, Parekh DJ, Cookson MS et al (2003) Randomized prospective evaluation of extended versus limited lymph node dissection in patients with clinically localized prostate cancer. J Urol 169:145-148

27. Aus G, Abbou CC, Bolla M et al (2005) EAU guidelines on prostate cancer. Eur Urol 48:546-551

28. Golimbu M, Provet J, Al-Askari S, Morales P (1987) Radical prostatectomy for stage D1 prostate cancer. Prognostic variables and results of treatment. Urology 30:427-435

29. Catalona WJ, Miller DR, Kavoussi LR (1988) Intermediateterm survival results in clinically understaged prostate cancer patients following radical prostatectomy. J Urol 140:540 543

30. Pound CR, Partin AW, Eisenberger MA, Chan DW, Pearson JD, Walsh PC (1999) Natural history of progression after PSA elevation following radical prostatectomy. JAMA 281(17):1591-1597 
31. Bader P, Burkhard FC, Markwalder R, Studer UE (2003) Disease progression and survival of patients with positive lymph nodes after radical prostatectomy. Is there a chance of cure? J Urol 169:849-854

32. Daneshmand S, Quek ML, Stein JP, Lieskovsky G, Cai J, Pinski J, Skinner EC, Skinner DG (2004) Prognosis of patients with lymph node positive prostate cancer following radical prostatectomy: long-term results. J Urol 172(6 Pt 1):22522255

33. Allaf ME, Palapattu GS, Trock BJ, Carter HB, Walsh PC (2004) Anatomical extent of lymph node dissection: impact on men with clinically localized prostate cancer J Urol 175(85 Pt 1):1840-1844

34. Bader P, Spahn M, Huber R, Echtle D, Frohneberg D (2004) Limited lymph node dissection in prostate cancer may miss lymph node metastases and determines outcome of apparently pN0 prostate cancer. Eur Urol 3(Suppl):16; abstract no. 55

35. Joslyn S, Konety B (2006) Impact of extent of lymphadenectomy on survival after radical prostatectomy for prostate cancer. Urology 68(1):121-125

36. Masterson TA, Bianco FJ Jr, Vickers AJ, DiBlasio CJ, Fearn PA, Rabbani F, Eastham JA, Scardino PT (2006) The association between total and positive lymph node counts, and disease progression in clinically localized prostate cancer. J Urol 175(4):1320-1324; discussion 1324-1325

37. Ferrari AC, Stone NN, Kurek R et al (2006) Molecular load of pathologically occult metastases in pelvic lymph nodes is an independent prognostic marker of biochemical failure after localized prostate cancer treatment. J Clin Oncol 24(19):3081-3088

38. Serni S, Masieri L, Minervini A, Lapini A, Nesi G, Carini M (2006) Cancer progression after anterograde radical prostatectomy for pathologic Gleason score 8 to 10 and influence of concomitant variables. Urology 67:373-378

39. Feinstein AR, Sosin DM, Wells CK (1985) The Will Rogers phenomenon. Stage migration and new diagnostic techniques as a source of misleading statistics for survival in cancer. $\mathrm{N}$ Engl J Med 312:1604-1608

40. DiMarco DS, Zincke H, Sebo TJ, Slezak J, Bergstralh EJ, Blute ML (2005) The extent of lymphadenectomy for pTXNO prostate cancer does not affect prostate cancer outcome in the prostate specific antigen era. J Urol 173(4):1121-1125

41. Schumacher M, Burkhard F, Thalmann G, Fleischmann A, Studer U. Is pelvic lymph node dissection necessary in patients with a serum PSA $<10 \mathrm{ng} / \mathrm{ml}$ undergoing radical prostatectomy for prostate cancer? Euro Urol 50(2):272-279

42. Grossfeld GD, Chang JJ, Broering JM, Li Y, Lubeck DP, Flanders SC et al (2001) Under staging and under grading in a contemporary series of patients undergoing radical prostatectomy: results from the cancer of the prostate strategic urologic research endeavour database. J Urol 165:851-856

43. Campbell SC, Klein EA, Levin HS, Piedmonte MR (1995) Open pelvic lymph node dissection for prostate cancer: a reassessment. Urology 46:352-355 